\title{
Correction to: Principles of Motor Learning to Support Neuroplasticity After ACL Injury: Implications for Optimizing Performance and Reducing Risk of Second ACL Injury
}

\author{
Alli Gokeler ${ }^{1,2,3}$ (D) Dorothee Neuhaus ${ }^{1} \cdot$ Anne Benjaminse ${ }^{3,4} \cdot$ Dustin R. Grooms $^{5,6}$ • Jochen Baumeister ${ }^{1,7,8}$
}

Published online: 21 February 2019

(c) Springer Nature Switzerland AG 2019

\section{Correction to: Sports Medicine} https://doi.org/10.1007/s40279-019-01058-0

Page 3, Section 2.1.1, sentence 4: This sentence, which previously read:

"As ACL injury may alter intracortical facilitation [34] and depressed intracortical inhibition is correlated with decreased quadriceps voluntary activation capability [35], external focus training may provide a means to restore quadriceps muscle activity via increasing intracortical inhibition." should read:

"As ACL injury may alter intracortical facilitation [34] and increased intracortical inhibition is correlated with decreased quadriceps voluntary activation capability [35], external focus training may provide a means to restore quadriceps muscle activity via depressing intracortical inhibition."
The original article can be found online at https://doi.org/10.1007/ s40279-019-01058-0.

\section{Alli Gokeler}

alli.gokeler@uni-paderborn.de

1 Exercise Science \& Neuroscience Unit, Department Exercise and Health, Faculty of Science, Paderborn University,

Paderborn, Germany

2 Luxembourg Institute of Research in Orthopedics, Sports Medicine and Science (LIROMS), Luxembourg, Luxembourg

3 Center for Human Movement Sciences, University of Groningen, University Medical Center Groningen, Groningen, The Netherlands

4 School of Sport Studies, Hanze University Groningen, Groningen, The Netherlands
5 Division of Athletic Training, School of Applied Health Sciences and Wellness, College of Health Sciences and Professions, Ohio University, Athens, OH, USA

6 Ohio Musculoskeletal and Neurological Institute, Ohio University, Athens, OH, USA

7 Exercise Neuroscience and Health Lab, Institute of Health, Nutrition and Sport Sciences, University of Flensburg, Flensburg, Germany

8 Division of Physiotherapy/Central Analytical Facilities (CAF) 3D Human Biomechanics Unit, Faculty of Medicine and Health, Stellenbosch University, Stellenbosch, South Africa 